\title{
On the Moment Generating Functions of Generalized order Statistics from General Class of Distributions and Characterizations
}

\author{
ELdesoky E. Afify* \\ Department of Mathematics, College Of Sciences and Arts, AL Qassim University, AL Maznab, Saudi Arabia
}

Received: 2 Mar. 2013, Revised: 3 Jun. 2013, Accepted: 5 Jun. 2013

Published online: 1 Sep. 2013

\begin{abstract}
In this paper, we establish some recurrence relations for both marginal and joint moment generating functions of generalized order statistics are established. The recurrence relations for both marginal and joint moment generating functions for order statistics and record values are deduced. We characterized a general class of distribution functions through conditional expectation,marginal and joint moment generating functions of generalized order statistics. Special cases are discussed.
\end{abstract}

Keywords: marginal and joint moment generating functions, generalized order statistics, recurrence relations, single moments, product moments, order statistics,record values, characterization.

AMS 2000 Subject Classification: 62G30

\section{Introduction}

The generalized order statistics (gOSs) have introduced by Kamps (1995a). Ordinary order statistics (oOSs), ordinary record values (oRVs), sequential order statistics and ordering via truncated distributions can be discussed as they are Special cases of gOSs. Balakrishnan and Ahsanullah (1994) obtained some recurrence for single and product moments of record values from Lomax distribution. Kamps (1995a, b, 1999) gave several recurrence relations for single and product moments of gOSs. Ahsanullah and Raqab (1999, 2000) have established recurrence relations for mariginal and joint moment generating functions from Gumbel and power distributions, respectively. Saran and Pandy (2003) gave some recurrence relations satisfied by marginal and joint moment generating functions of generalized order statistics from power function distribution. Haseeb and Hasan (2004) obtained some recurrence relations for single and product moments of generalized order statistics from general class of distribution $1-F(x)=[a h(x)+b]^{c}$. Abd EL Baset (2007) obtained some recurrence relations for single and product moments of generalized order statistics from doubly truncated Burr type XII distribution. Bakheet and Jawa (2008) obtained a recurrence relation of single and product moments of generalized order statistics from general class of distributions $1-F(x)=[a h(x)+b]^{c}$. Khan, et al (2010) characterized a general form of distribution functions $F(x)=[a h(x)+b]^{c}$ through conditional expectation of dual generalized order statistics. Shawky and Bakoban (2009)characterized general class of distributions by considering the conditional expectation of record values. Kumar and Khan (2013) obtained some recurrence relations satisfied by single and product moments of gOS from doubly truncated generalized exponential distribution, they characterized the distribution using a recurrence relation of single moments. In this paper, some recurrence relations for both marginal and joint moment generating functions of generalized order statistics are established. The recurrence relations for both marginal and joint moment generating functions for order statistics and record values are deduced. We characterize a general class of distribution functions through conditional expectation, marginal and joint moment generating functions of generalized order statistics. Special cases are discussed. Suppose that the random variable $\mathrm{X}$ has a distribution function (df) of the following class of absolutely continuous distribution

$$
1-F(x)=\left[a x^{p}+b\right]^{c}
$$

\footnotetext{
* Corresponding author e-mail: eldesoky@yahoo.com
} 
and probability density function (pdf)

$$
f(x)=-a c p x^{c-1}\left(a x^{p}+b\right)^{c-1}
$$

From (1.1) and (1.2) we get

$$
\left(b+a x^{p}\right) f(x)=-a c p x^{p-1}[1-F(x)]
$$

Suppose $\mathrm{X}(1, \mathrm{n}, \mathrm{m}, \mathrm{k}), \mathrm{X}(2, \mathrm{n}, \mathrm{m}, \mathrm{k}), \mathrm{X}(\mathrm{n}, \mathrm{n}, \mathrm{m}, \mathrm{k}), k \geq 1$ and $\mathrm{m}$ being a real number, are $\mathrm{n}$ generalized order statistics based on an absolutely continuous cumulative distribution function (cdf) $\mathrm{F}(\mathrm{x})$ with probability density function (pdf) $f(x)$, then they having joint density function of the form (Kamps 1995b)

$$
\begin{gathered}
f_{X(1, n, m, k), \ldots, X(n, n, m, k)}\left(x_{1}, \ldots, x_{n}\right)= \\
k\left(\prod_{j=1}^{n-1} \gamma_{j}\right)\left(\prod_{i=1}^{n-1}\left[1-F\left(x_{i}\right)\right]^{m_{i}} f\left(x_{i}\right)\right)\left[1-F\left(x_{n}\right)\right]^{k-1} f\left(x_{n}\right)
\end{gathered}
$$

The pdf of generalized order statistics $X_{(r, n, m, k)}$ is given by ( Kamps 1995b)

$$
\begin{aligned}
& f_{r, n, m, k}(x)=\frac{C_{r-1}}{(r-1) !} f(x)[1-F(x)]^{\gamma_{r}-1} g_{m}^{r-1}[F(x)], \\
& F^{-1}(0)<x<F^{-1}(1)
\end{aligned}
$$

where

$C_{r-1}=\prod_{j=1}^{r} \gamma_{j}, \gamma_{j}=k+(n-j)(m+1), j=1,2, \ldots, n$, and for $x \varepsilon(0,1)$, and for $0<x<1$

$$
g_{m}(x)= \begin{cases}\frac{1}{m+1}\left[1-(1-x)^{m+1}\right], & m \neq-1 \\ -\ln (1-x), & m=-1\end{cases}
$$

Similarly, the joint pdf of the r-th generalized order statistics $X_{r, n, m, k}$ and s-th generalized order statistics $X_{s, n, m, k}$ for $1 \leq r<s \leq n$ is given by and the joint pdf of $\mathrm{X}(\mathrm{r}, \mathrm{n}, \mathrm{m}, \mathrm{k})$ and $\mathrm{X}(\mathrm{s}, \mathrm{n}, \mathrm{m}, \mathrm{k}), 1 \leq r<s \leq n$ is given by kamps (1995) as follows

$$
\begin{gathered}
f_{X(r, n, m, k), X(s, n, m, k)}(x, y)=\frac{C_{s-1}}{(r-1) !(s-r-1) !} f(x) f(y) \\
{[1-F(x)]^{m} g_{m}^{r-1}(F(x))\left[g_{m}(F(y))-g_{m}(F(x))\right]^{s-r-1}[1-F(y)]^{\gamma_{r}-1},}
\end{gathered}
$$

For $1 \leq r<s \leq n$, the conditional pdf of $X_{(s, n, m, k)}$ given $X_{(r, n, m, k)}=X$ is

$$
\frac{\gamma_{r+1}}{(r-1) !}[1-F(x)]^{m-\gamma_{r}+1}\left[g_{m}(F(y))-g_{m}(F(x))\right]^{s-r-1}[1-F(y)]^{\gamma_{s}-1} f(y),
$$
is

and the conditional pdf of $X_{(r, n, m, k)}$ given $X_{(s, n, m, k)}=y$

$$
\begin{aligned}
& \frac{(s-1) !}{(r-1) !(s-r-1) !} f(x) g_{m}^{r-1}(F(x))[1-F(x)]^{m} g_{m}^{1-s}(F(x)) \\
& {\left[g_{m}(F(y))-g_{m}(F(x))\right]^{s-r-1}}
\end{aligned}
$$

Obtaining moment generating functions (MGF) of the gOS allow us to generate the MGF's and then the moments of the entire sequence of gOS's, the estimation of the parameters involved in the parent distribution and prediction of the future can be derived based on the moments. Let us denote the marginal moment generating of $X_{r, n, m, k)}$ by $M_{X(r, n, m, k}(t)$ and its $\mathrm{j}$-th derivative by $M_{X(r, n, m, k)}^{(j)}$. Similarly, let $M_{X(r, s, n, m, k)}\left(t_{1}, t_{2}\right)$ denote the joint moment generating function of $\left.X_{(} r, n, m, k\right)$ and $X_{(s, n, m, k)}$ and its $\mathrm{i}, \mathrm{j}$-th partial derivative by $M_{X(r, s, n, m, k)}^{(i, j)}\left(t_{1}, t_{2}\right)$.

\section{Recurrence Relations}

\subsection{Marginal Recurrence Relations}

The MGF of the r-th $\mathrm{gOS} X_{(r, n, m, k)}$ is given by

$$
M_{r, n, m, k}(t)=\frac{C_{r-1}}{(r-1) !} \int_{0}^{\infty} e^{t x} f(x)[1-F(x)]^{\gamma_{r}-1} g_{m}^{r-1}[F(x)] d x
$$

where $f(x)$ and $F(x)$ are given in equations (1.1) and (1.2) respectively. Then by making use of the characterization differential equation (1.3), we establish in this section some recurrence relations for marginal moments of generalized order statistics.

\section{Theorem 1:}

For $\mathrm{i}=1,2, \ldots$, and $1 \leq r<s \leq n-1$, we have

$$
\begin{array}{r}
M_{r+1, n, m, k}(t)=M_{r, n, m, k}(t) \\
-\frac{t}{c p \gamma_{r}} M_{r+1, n, m, k}^{(1)}-\frac{b t}{c p \gamma_{r+1}} M_{r+1, n, m, k}^{(1-p)}
\end{array}
$$

Proof: From (2.1)

$$
M_{r+1, n, m, k}(t)=\frac{C_{r}}{r !} \int_{0}^{\infty} e^{t x} f(x)[1-F(x)]^{\gamma_{r+1}} g_{m}^{r}[F(x)] d x
$$

Integrating by parts treating $e^{t x} g_{m}^{r}[F(x)]$ for differention and the rest of integrand for integration, we have

$$
\begin{gathered}
M_{r+1, n, m, k}(t)=\frac{C_{r}}{(r) !} \int_{0}^{\infty} e^{t x} f(x)[1-F(x)]^{\gamma_{r-1}} g_{m}^{r-1}[F(x)] d x \\
+\frac{t C_{r}}{\gamma_{r+1} r !} \int_{0}^{\infty} e^{t x} f(x)[1-F(x)]^{\gamma_{r+1}} d x
\end{gathered}
$$

Using (1.3) for the second part of the right hand side, we have

$$
\begin{gathered}
M_{r+1, n, m, k}(t)=M_{r, n, m, k}(t) \\
-\frac{t C_{r}}{a c p \gamma_{r+1} r !} \int_{0}^{\infty} e^{t x}\left(a x+b x^{1-p}\right) f(x)[1-F(x)]^{\gamma_{r+1}-1} g_{m}^{r}[F(x)] d x
\end{gathered}
$$

Simplifying the above equation, we obtain (2.2). Differentiating (2.2) $(\mathrm{j}+\mathrm{p})$ times with respect to $t$ then setting $\mathrm{t}=0$, we get 


$$
\begin{gathered}
\mu_{r+1, n, m, k}^{j+p}=\frac{c p \gamma_{r+1}}{c p \gamma_{r+1}+j+p} \mu_{r+1, n, m, k}^{j+p} \\
-\frac{b(b+j)}{a\left[c p \gamma_{r+1}+j+p\right]} \mu_{r+1, n, m, k}^{j}
\end{gathered}
$$

\subsection{Joint Recurrence Relations}

The joint MGF of $X_{r, n, m, k}$ and $X_{s, n, m, k}, 1 \leq r<s \leq n$ is given

$$
M_{r, s, n, m, k}\left(t_{1}, t_{2}\right)=\frac{C_{s-1}}{(r-1) !(s-r-1) !} \int_{0}^{\infty} \int_{y=x}^{\infty} e^{t_{1} x+t_{2} y} f(x) f(y)
$$$$
[1-F(x)]^{m} g_{m}^{r-1}[F(x)]\left[g_{m}(F(y))-g_{m}(F(x))\right]^{s-r-1}[1-F(x)]^{\gamma_{s}-1} d y d x \quad(2.4)
$$

By use of (1.3), we establish in this section some recurrence relations for joint moments of generalized order statistics.

Theorem 2: For $\mathrm{i}=1,2, \ldots$, and $1 \leq r<s \leq n-1$, we have

$$
\begin{gathered}
M_{r, s+1, n, m, k}\left(t_{1}, t_{2}\right)=M_{r, s, n, m, k}\left(t_{1}, t_{2}\right) \\
-\frac{t_{2}}{c p \gamma_{r+1}} M_{r, s+1, n, m, k}^{(0,1)}\left(t_{1}, t_{2}\right)-\frac{b t_{2}}{a c p \gamma_{r+1}} M_{r, s+1, n, m, k}^{(0,1-p)}\left(t_{1}, t_{2}\right)(2.5)
\end{gathered}
$$

Proof: From (2.4), we have

$$
\begin{array}{r}
M_{r, s+1, n, m, k}\left(t_{1}, t_{2}\right)=\frac{C_{s}}{(r-1) !(s-r) !} \\
\int_{x=0}^{\infty} e^{t_{1} x} f(x)[1-F(x)]^{m} g_{m}^{r-1}[F(x)] I(x) d x
\end{array}
$$

where

$I(x)=\int_{y=x}^{\infty} e^{t_{2} y} f(y)\left[g_{m}(F(y))-g_{m}(F(x))\right]^{s-r}[1-F(y)]^{\gamma_{s+1}-1} d y$

Integrating $\mathrm{I}(\mathrm{x})$ by parts treating $e^{t_{2} y}\left[g_{m}[F(y)]-g_{m}[F(x)]^{s-r}\right.$ for differention and the rest of integrand for integration, we have

$$
\begin{aligned}
& I(x)=\frac{s-r}{\gamma_{r+1}} \int_{y=x}^{\infty} e^{t_{2} x} f(y)\left[g_{m}(F(y))-g_{m}(F(x))\right]^{s-r-1}[1-F(y)]^{\gamma_{s+1}} d y \\
& +\frac{t_{2}}{\gamma_{r+1}} \int_{y=x}^{\infty} e^{t_{2} y}\left[g_{m}(F(y))-g_{m}(F(x))\right]^{s-r}[1-F(y)]^{\gamma_{s+1}} d y
\end{aligned}
$$

Substituting from (2.7) into (2.6) we have

$$
\begin{gathered}
M_{r, s+1, n, m, k}\left(t_{1}, t_{2}\right)=\frac{C_{s}}{\gamma_{s+1}(r-1) !(s-r-1) !} \\
\int_{0}^{\infty} \int_{y=x}^{\infty} e^{t_{1} x+t_{2} y} f(x) f(y)[1-F(x)]^{m} \\
g_{m}^{r-1}[F(x)]\left[g_{m}(F(y))-g_{m}(F(x))\right]^{s-r-1}[1-F(y)]^{\gamma_{s+1}-1} d y d x \\
+\frac{t_{2} C_{s}}{\gamma_{s+1}(r-1) !(s-r) !} \int_{0}^{\infty} \int_{y=x}^{\infty} e^{t_{1} x+t_{2} y} f(x)[1-F(x)]^{m}
\end{gathered}
$$

$$
\begin{gathered}
g_{m}^{r-1}[F(x)]\left[g_{m}(F(y))-g_{m}(F(x))\right]^{s-r}[1-F(y)]^{\gamma_{s+1}} d y d x \\
M_{r, s+1, n, m, k}\left(t_{1}, t_{2}\right)=M_{r, s, n, m, k}\left(t_{1}, t_{2}\right) \\
-\frac{t_{2} C_{s}}{a c p \gamma_{s+1}(r-1) !(s-r) !} \int_{0}^{\infty} \int_{y=x}^{\infty}\left(a y+b y^{1-p}\right) e^{t_{1} x+t_{2} y} f(x) f(y)[1-F(x)]^{m} \\
g_{m}^{r-1}[F(x)]\left[g_{m}(F(y))-g_{m}(F(x))\right]^{s-r}[1-F(y)]^{\gamma_{s+1}-1} d y d x
\end{gathered}
$$

Simplifying the above equation we get (2.5). Differentiating (2.5) i times with respect to $t_{1}$ and $(\mathrm{j}+\mathrm{p})$ times with respect to $t_{2}$ then setting $t_{1}=t_{2}=0$, we get the following equation

$$
\mu_{r, s+1, n, m, k}^{i, j+p}=\frac{-b(b+j)}{a\left[c p \gamma_{r+1}+j+p\right]} \mu_{r, s+1, n, m, k}^{i, j}
$$

$+\frac{c p \gamma_{r+1}}{c p \gamma_{r+1}+j+p} \mu_{r, s, n, m, k}^{i, j+p}$

\section{a) Remarks:}

1) Setting $b=1$ and $c=-d$ in (2.2) and (2.5), we obtain the marginal and joint recurrence relations of gOS from Burr XII distribution.

2) Setting $b=1$ and $c=-d$ in (2.3) and (2.8), we obtain the recurrence relations for single and product moments of gOS from Burr XII distribution, see Abd El Baset (2007) in non truncated case.

3) Setting $\mathrm{a}=\mathrm{b}=\mathrm{p}=1$ and $c=-\gamma$ in (2.2) and (2.5), we get the marginal and joint recurrence relations of gOS from Lomax distribution.

4) Setting $\mathrm{a}=\mathrm{b}=\mathrm{p}=1$ and $c=-\gamma, \mathrm{m}=-1$ and $\mathrm{k}=1$ in (2.3) and (2.8) we obtain the recurrence relations for record values from Lomax distribution, see Balakrishnan and Ahsanuallah (1994).

5) Setting $a=-1, c=1$ and $b=1$ in (2.2) and (2.4), we obtain the marginal and joint recurrence relations of $\mathrm{gOS}$ from from power function distribution (see Abd Al Baset 2007).

6) Setting $a=-1, c=1, b=1 \mathrm{~m}=0$ and $\mathrm{k}=1$ in (2.3) and (2.8), single and product moments of OS from Pareto distribution (non- truncated case) were obtained by Khurana and Jha (1995).

\section{Characterizations}

This section contains a characterization of general class of distributions which can characterize the special cases distributions by considering the corresponding parameters, based on the proposition of Lin (1986). 


\section{Theorem 4.1}

The necessary and sufficient condition for a random variable $\mathrm{X}$ to be distributed with pdf given by (1.1) is that

$$
\begin{array}{r}
M_{r+1, n, m, k}(t)=M_{r, n, m, k}(t) \\
-\frac{t}{c p \gamma_{r}} M_{r+1, n, m, k}^{(1)}-\frac{b t}{c p \gamma_{r+1}} M_{r+1, n, m, k}^{(1-p)}
\end{array}
$$

Proof: A necessary part follows immediately from (2.1). on the other hand if the recurrence relation (4.1) is satisfied, then

$$
\begin{gathered}
\frac{C_{r}}{r !} \int_{0}^{\infty} e^{t x} f(x)[1-F(x)]^{\gamma_{r+1}-1} g_{m}^{r}(F(x)) d x= \\
\frac{C_{r-1}}{(r-1) !} \int_{0}^{\infty} e^{t x} f(x)[1-F(x)]^{\gamma_{r}-1} g_{m}^{r-1}(F(x)) d x \\
-\frac{t C_{r}}{c p \gamma_{r+1} r !} \int_{0}^{\infty} x e^{t x} f(x)[1-F(x)]^{\gamma_{r+1}-1} g_{m}^{r}(F(x)) d x \\
-\frac{b t C_{r}}{a c p \gamma_{r+1} r !} \int_{0}^{\infty} e^{t x} x^{1-p} f(x)[1-F(x)]^{\gamma_{r+1}-1} g_{m}^{r}(F(x)) d x
\end{gathered}
$$

Integrating the first part on the right hand side by parts treating $e^{t x}[1-F(x)]^{\gamma_{r+1}}$ for differentiation and the rest of integrand for integration, we get after simplification that

$$
\begin{gathered}
\frac{t C_{r}}{a c p \gamma_{r+1} r !} \int_{0}^{\infty} e^{t x} g_{m}^{r}(F(x)) \\
{[1-F(x)]^{\gamma_{r+1}-1}\left[-[1-F(x)]-\frac{1}{c p} x f(x)-\frac{b x^{1-p}}{a c p} f(x)\right] d x=0}
\end{gathered}
$$

It now follows from proposition of Lin (1986) that

$$
f(x)=-a c p x^{c-1}\left(a x^{p}+b\right)^{c-1}
$$

which proves that $\mathrm{f}(\mathrm{x})$ has the form (1.1).

\section{Theorem 4.2}

In this section, we characterize the general class of distribution recurrence relation of joint moments. The necessary and sufficient condition for a random variable $\mathrm{X}$ to be distributed with pdf given by (1.1) is that

$$
\begin{gathered}
M_{r, s+1, n, m, k}\left(t_{1}, t_{2}\right)=M_{r, s, n, m, k}\left(t_{1}, t_{2}\right) \\
-\frac{t_{2}}{c p \gamma_{r+1}} M_{r, s+1, n, m, k}^{(0,1)}\left(t_{1}, t_{2}\right)-\frac{b t_{2}}{a c p \gamma_{r+1}} M_{r, s+1, n, m, k}^{(0,1-p)}\left(t_{1}, t_{2}\right)
\end{gathered}
$$

Proof: A necessary part follows immediately from (2.5). on the other hand if the recurrence

$$
\frac{C_{s}}{(r-1) !(s-r) !} \int_{0}^{\infty} \int_{y=x}^{\infty} e^{t_{1} x+t_{2} y} f(x) f(y)[1-F(x)]^{m}
$$

$$
\begin{aligned}
& {\left[g_{m}(F(y))-g_{m}(F(x))\right]^{s-r}[1-F(x)]^{\gamma_{r+1}-1} g_{m}^{r-1}(F(x)) d y d x} \\
& =\frac{C_{s-1}}{(r-1) !(s-r-1) !} \int_{x=0}^{\infty} \int_{y=x}^{\infty} e^{t_{1} x} f(x)[1-F(x)]^{m} g_{m}^{r-1}(F(x)) \\
& {[1-F(y)]^{\gamma_{s}-1} I(x) d x} \\
& -\frac{t_{2} C_{s}}{c p \gamma_{s+1}(r-1) !(s-r) !} \int_{x=0}^{\infty} \int_{y=x}^{\infty} e^{t_{1} x+t_{2} y} y f(x) f(y) \\
& {[1-F(x)]^{m} g_{m}^{r-1}(F(x))} \\
& {\left[g_{m}(F(y))-g_{m}(F(x))\right]^{s-r}[1-F(y)]^{\gamma_{s+1}-1} d y d x} \\
& -\frac{b t_{2} C_{s}}{c p \gamma_{s+1}(r-1) !(s-r) !} \int_{x=0}^{\infty} \int_{y=x}^{\infty} e^{t_{1} x+t_{2} y} y^{1-} f(x) f(y)[1-F(x)]^{m} g_{m}^{r-1}(F(x)) \\
& {\left[g_{m}(F(y))-g_{m}(F(x))\right]^{s-r}[1-F(y)]^{\gamma_{s+1}-1} d y d x}
\end{aligned}
$$

where

$I(x)=\int_{y=x}^{\infty} e^{t_{2} y} f(y)\left[g_{m}(F(y))-g_{m}(F(x))\right]^{s-r-1}[1-F(x)]^{\gamma_{s+1}} d y$

Integrating $\mathrm{I}(\mathrm{x})$ by parts treating $e^{t_{2} y}[1-F(y)]^{\gamma_{s+1}}$ as a part for differentiation and the rest of integrand as the part of integration then substitute in (4.3) and simplify we get

$$
\begin{gathered}
-\frac{t_{2} C_{s}}{\gamma_{s+1}(r-1) !(s-r) !} \int_{x=0}^{\infty} \int_{y=x}^{\infty} e^{t_{1} x+t_{2} y} f(x) \\
{[1-F(x)]^{m} g_{m}^{r-1}[F(x)]} \\
{\left[g_{m}(F(y))-g_{m}(F(x))\right]^{s-r}[1-F(y)]^{\gamma_{s+1}-1}} \\
{\left[[1-F(y)]+\frac{y f(y)}{c p}+\frac{b y^{1-p} f(y)}{a c p}\right] d y d x=0}
\end{gathered}
$$

It now follows from proposition of Lin (1986) that

$$
\left(b+a y^{p}\right) f(x)=-a c p y^{p-1}[1-F(y)]
$$

which proves that $\mathrm{f}(\mathrm{x})$ has the form (1.1).

\section{Theorem 4.3}

Let $X_{(1, n, m, k)}, X_{(2, n, m, k)}, \ldots, X_{(n, n, m, k)}$ be generalized order statistics based on continuous distribution function F. Then the conditional expectation of generalized order statistics $X_{(r+1, n, m, k)}$ given $X_{(r, n, m, k)}$ is given by

$$
\begin{gathered}
E\left(X_{r+1, n, m, k} / X_{r, n, m, k}=x\right)=x[1-F(x)]^{m-\gamma_{r}+\gamma_{r+1}+1} \\
+[1-F(x)]^{m-\gamma_{r}+1} \sum_{j=0}^{c \gamma_{r+1}}\left(\begin{array}{c}
c \gamma_{r+1} \\
j
\end{array}\right) \frac{b^{c \gamma_{r+1}-1}}{p j+1}\left[\beta^{p j+1}-x^{p j+1}\right]
\end{gathered}
$$


iff $\mathrm{X}$ has a general class distribution of (1.1).

\section{Proof:} (1.1).

First, we assume that $\mathrm{X}$ has general class distribution

From (1.7) with $\mathrm{s}=\mathrm{r}+1$, we have

$$
\begin{gathered}
E\left(X_{r+1, n, m, k} / X_{r, n, m, k}=x\right)=\gamma_{r+1}[1-F(x)]^{m-\gamma_{r}+1} \\
\int_{x}^{\infty} y f(y)[1-F(y)]^{\gamma_{s+1}-1} d y
\end{gathered}
$$

Integrating by parts treating y for differentiation and the rest of integrand for integration, we obtain

$$
\begin{gathered}
E\left(X_{r+1, n, m, k} / X_{r, n, m, k}=x\right)=x[1-F(x)]^{m-\gamma_{r}+\gamma_{r+1}+1} \\
+[1-F(x)]^{m-\gamma_{r}+1} \int_{x}^{\infty}[1-F(y)]^{\gamma_{s+1}} d y
\end{gathered}
$$

Using (1.2) and by using binomial expansion we obtain

$$
\begin{aligned}
& E\left(X_{r+1, n, m, k} / X_{r, n, m, k}=x\right)=x[1-F(x)]^{m-\gamma_{r}+\gamma_{r+1}+1} \\
& +[1-F(x)]^{m-\gamma_{r}+1} \sum_{j=0}^{c \gamma_{r+1}}\left(\begin{array}{c}
c \gamma_{r+1} \\
j
\end{array}\right) b^{c \gamma_{r+1}} a^{j} \int_{x}^{\infty} y^{p j} d y
\end{aligned}
$$

which leads easily to (4.1).

Second, to prove (4.4) implies $\mathrm{X}$ has general class distribution (1.1), we have

$$
\begin{gathered}
\gamma_{r+1} \int_{x}^{\infty} y f(y)[1-F(y)]^{\gamma_{r+1}-1} d y=x[1-F(y)]^{\gamma_{r+1}} \\
+\sum_{j=0}^{c \gamma_{r+1}}\left(\begin{array}{c}
c \gamma_{r+1} \\
j
\end{array}\right) \frac{a^{j} b^{c \gamma_{r+1}-1}}{p j+1}\left[\beta^{p j+1}-x^{p j+1}\right]
\end{gathered}
$$

Differentiating sides w. r. t. $\mathrm{x}$ and simplifying we have both

$$
[1-F(y)]^{\gamma_{r+1}}=\left[\left(a x^{p}+b\right)^{c}\right]^{\gamma_{r+1}}
$$

Therefore

$$
1-F(x)=\left(a x^{p}+b\right)^{c}
$$

\section{Remarks}

1) Under the assumption of theorem (4.1), (4.2) and (4.3) recurrence relations characterize the special cases distributions from the general class of distributions by choosing the corresponding appropriate parameters in (4.1), (4.2) and (4.3).

2) Under the assumption of theorem (4.1), (4.2) and (4.3) with $\mathrm{m}=0, \mathrm{k}=1$ and proper chose of $\mathrm{a}, \mathrm{b}, \mathrm{c}$ and $\mathrm{p}$, we obtain the order statistics case, see Khan and Abouammoh (1999).

3) Setting $m=-1$ and $k=1$ in (4.1), (4.2) and (4.3) we obtain the record case.

\section{Applications}

Recurrence relations are useful in reducing the number of operations necessary to obtain a general form for the function under consideration. Furthermore, they are used in characterization distributions, which in important area, permitting the identification of population distribution from the properties of the sample. The results established in this paper can be used to determine the mean, variance and coefficients of skewness and korosis. The moments can also used for finding best linear unbiased estimator (BLUE) for parameter and conditional moments. we are planning to estimate the parameters of a special case of the general class of distributions by maximum likelihood method and comparing it by (BLUE).

\section{Conclusion}

This paper deals with the generalized order statistics form general class of distributions Two characterizing results of general class of distributions have been obtained using marginal and joint moment generating and conditional expectation of generalized order statistics. Special cases were deduced.

\section{References}

[1] Abd El Baset A. A. Recurrence relationsfor single and product moments of generalized order statistics from doubly truncated Burr type XII distribution. Journal Egypt. Math. Soc., 117-128 (2007).

[2] Ahsanullah, M. Recor Statistics. Nova Science Publishers, Inc, Commack, NY, USA, (1995).

[3] Ahsanullah, M. and Raqab, M. Z. Recurrence relations for moment generating functions of record values from Pareto and Gumbel distributions, Stochastic Modeling and Applictions, 2, 35-48 (1999)

[4] Bakheet N. A. and Jawa, M. T. Recurrence relations for single moments of generalized order statistics from doubly truncated continuous distributions. JKAU: Sci., 20, 79-89 (2008).

[5] Balakrishnan, N. and Ahsanullah, M. Relations for single and product moments of record values from Lomax distribution. Sankhya, 56, 140-146 (1994).

[6] Devendra Kumar and M. I. khan. Relations for generalized order statistics from doubly truncated generalized exponential distribution and its characterization. Math. Sci. Lett., 2, 9-18 (2013).

[7] Haseeb, A. and Hasan, M. I. Recurrence relationsfor single and product moments of generalized order statistics from a general class of distribution. Metron, LXII, 327-337 (2004).

[8] Khan, A. H. and Abouammoh, A. M. Characterizations of distributions by conditional expectation of order statistics, 9, 159-168 (1999).

[9] Khan, A. H.,Zaki Anwer and Safia Chishti Characterization of continuous distributions through conditional expectation of functions of dual generalized order statistics. Pak. j. of Statistics, 26, 615-628 (2010). 
[10] Khurana, A. P. and Jha, V. D. Some recurrence relations between product moments of order statistics of a doubly truncated Pareto distribution. Sankhya, 57, 1-9 (1995).

[11] Raqab, M. Z. and Ahsanullah, M. Relations for marginal and joint moment generating functions of record values from Power function distribution. Journal of Applied Statistical Science, 10, 27-36 (2000).

[12] Saran, J. and Pandey, A. Recurrence relations for marginal and joint moment generating functions of generalized order statistics from power function distribution. Metron, LX1, 27-33 (2003).

[13] Shawky,A. I. and Bakoban, R. A. Conditional expectation of certain distributions of record values. Int. Journal of Math. Analysis, 3, 829-838 (2009).

[14] Khurana, A. P. and Jha, V. D. Some recurrence relations between product moments of order statistics of a doubly truncated Pareto distribution. Sankhya, 57, 1-9 (1995). 